\title{
X-RAY PROPERTIES OF LINERS AND LOW LUMINOSITY SEYFERT GALAXIES OBSERVED WITH ASCA
}

\author{
Y.TERASHIMA, H.KUNIEDA \\ Department of Physics, Nagoya University, Chikusa-ku, Nagoya, \\ Japan \\ AND \\ P.J.SERLEMITSOS, A.PTAK \\ Code 662, NASA/Goddard Space Flight Center, Greenbelt, \\ $M D$ 20771, USA
}

\begin{abstract}
.
We present $A S C A$ spectra of low luminosity AGNs with X-ray luminosities of $10^{40-41} \mathrm{ergs} \mathrm{s}^{-1}$. Their X-ray continua are very similar to Seyfert galaxies. Although iron $\mathrm{K}$ emissin centered at $6.4 \mathrm{keV}$ or $6.7 \mathrm{keV}$ is detected from a few objects, iron $\mathrm{K}$ emission is weak on average. Possible reasons for variety of iron line properties are discussed.
\end{abstract}

\section{Observations and Results}

We compiled LINERs (Low Ionization Nuclear Emission-line Regions) and low luminosity Seyfert galaxies from which X-ray emission is considered to be dominated by low luminosity AGNs (LLAGNs): NGC 1097, NGC 3031, NGC 3998, NGC 4450, NGC 4579, NGC 4594, and NGC 5033. These galaxies have a X-ray nucleus, larger $\mathrm{X}$-ray to $\mathrm{B}$ band luminosity ratio than normal galaxies, and similar X-ray to $\mathrm{H} \alpha$ luminosity ratio to Seyfert galaxies.

Their X-ray continuum shape are very similar to Seyfert 1 galaxies; photon index $\sim 1.8$ with small absorption $\left(<10^{21} \mathrm{~cm}^{-2}\right)$. Iron $\mathrm{K}$ emission is detected from NGC 3031 [1][4], NGC 4579 [5], and NGC 5033. The line center energy is $6.7 \mathrm{keV}$ for NGC 3031 and NGC 4579 , and $6.4 \mathrm{keV}$ for NGC 5033. Other galaxies (NGC 1097, NGC 3998, NGC 4450, NGC 4594) 

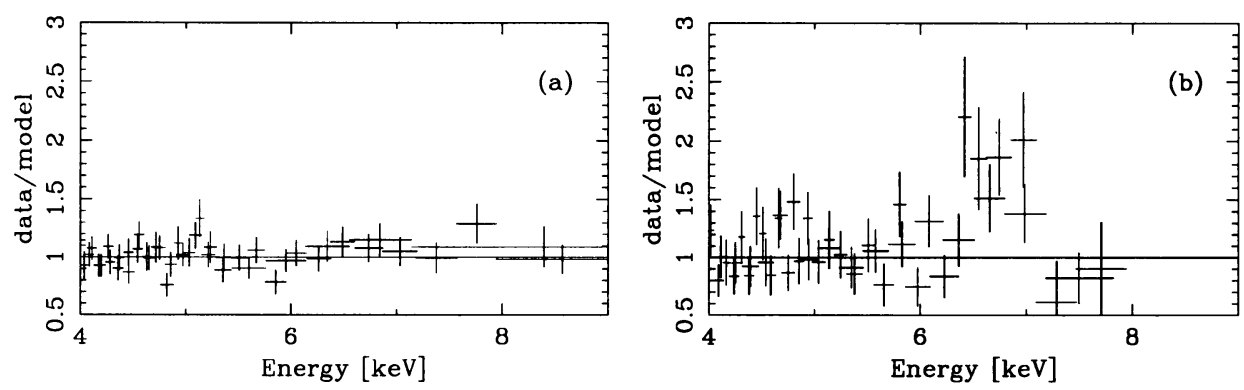

Figure 1. Data / continuum model around iron emission lines (a)averaged spectrum of NGC 1097, NGC 3998, NGC 4450, and NGC 4594 (b)NGC 4579

show no significant iron line (upper limit of an equivalent width $\mathrm{EW}=$ 200-300 eV at $90 \%$ confidence for one parameter). Since these LLAGNs have very similar X-ray characteristics, we summed up their X-ray spectra and made a composite spectrum. No significant iron emission line is seen in the composite spectrum and an upper limit of an equivalent width is $\sim 100$ $\mathrm{eV}$ for a narrow line at $6.4 \mathrm{keV}$ or $6.7 \mathrm{keV}$.

\section{Discussion}

The line center energies of NGC 3031 and NGC $4579(6.7 \mathrm{keV})$ are consistent with He-like iron and significantly higher than usual Seyfert 1s. An upper limit on the iron line equivalent width of the composite spectrum is weaker than Seyfert 1s. If iron lines are emitted from the accretion disk around the central blackhole, possible explanations of variety of the iron line properties are difference of inclination angle and/or ionization state of the accretion disk. If inclination angle becomes large, the line becomes very broad and hard to be detected. The blue shifted component from inclined disk may also explain higher line center energy. Alternatively, if the disk is ionized, the line center energy increases. When the ionization state of iron lies in FeXVII-FeXXIII, iron emission is suppressed since effective fluorescence yield is small due to resonant trapping [3] [6]. A possible problem is to maintain high ionization parameter under very low luminosity.

\section{References}

1. Ishisaki et al. 1996, PASJ, 48, 237

2. Makishima et al. 1994, PASJ, 46, L77

3. Ross \& Fabian 1993, MNRAS, 261, 74

4. Serlemitsos, Ptak, \& Yaqoob 1996, in Physics of LINERs in view of Recent Observations, eds. Eracleous et al.

5. Terashima et al. 1997 , in preparation

6. Życki \& Czerny 1994, MNRAS, 266, 653 